\title{
A two-year study of diffused retinal pigment epitheliopathy treated with half-dose photodynamic therapy guided by simultaneous angiography and optical coherence tomography
}

\author{
Yang $\mathrm{Liu}^{1,2} \cdot{\text { Lei } \mathrm{Li}^{1,2} \cdot \text { Elena Yingqiu } \mathrm{Zhu}^{3} \cdot \text { Yuanzhi Yuan }}^{4} \cdot$ Wenji Wang ${ }^{1,2} \cdot \mathrm{Gezhi} \mathrm{Xu}^{1,2}$
}

Received: 27 March 2018 / Revised: 18 September 2018 / Accepted: 4 November 2018 / Published online: 7 December 2018

(c) The Author(s) 2018. This article is published with open access

\begin{abstract}
Objectives Diffused retinal pigment epitheliopathy (DRPE) is not necessarily the same as chronic central serous chorioretinopathy (CSC), but a severe subgroup under the umbrella of chronic CSC. This study was to evaluate the efficacy and safety of half-dose PDT treating DRPE.

Methods A retrospective case series design was used. Forty-eight consecutive patients (48 eyes) with DRPE treated with half-dose PDT underwent follow-up at baseline, 3 months, 6 months, 12 months and 24 months. Simultaneous FA, ICGA and OCT were used for the treatment and follow-up. The primary outcomes were the subretinal fluid and best-corrected visual acuity in optical coherence tomography.

Results Complete fluid absorption was achieved in $95.8 \%$ of eyes at 3 months and $100.0 \%$ of eyes at 24 months. The baseline logarithm of the minimum angle of resolution (logMAR) BCVA, which was $0.51 \pm 0.36$, significantly improved to $0.43 \pm 0.38(p<0.001)$ at 6 months. The boost continued to $0.29 \pm 0.37(p<0.001)$ at 12 months and $0.19 \pm 0.39(p<0.001)$ at 24 months. The integrities of the ellipsoid zone (EZ) and interdigitation zone (IZ) improved throughout. Regression analyses showed the BCVA in logMAR was inversely correlated with the EZ $(p<0.01)$ and IZ $(p<0.01)$. The recurrence rate was $6.3 \%$. No severe complications were witnessed.

Conclusions In 48 eyes with DRPE, simultaneous angiography and OCT facilitated a more comprehensive guidance for half-dose PDT treatment and follow-up. The BCVA improvement occurred at 6 months, which may be attributed to the restoration of the outer retinal structure.
\end{abstract}

These authors contributed equally: Lei Li, Gezhi Xu

Lei Li

drlilei3028@163.com

$\triangle$ Gezhi Xu

16111260005@fudan.edu.cn

1 Department of Ophthalmology, Eye and ENT Hospital of Fudan University, Shanghai 200032, China

2 Shanghai Key Laboratory of Visual Impairment and Restoration, Eye and ENT Hospital, Shanghai Medical College, Fudan University, Shanghai 200032, China

3 Melbourne Medical School, University of Melbourne, Parkville, VIC 3010, Australia

4 Department of Ophthalmology, Zhong Shan Hospital of Fudan University, Shanghai 200032, China

\section{Introduction}

Central serous chorioretinopathy (CSC) is defined as a serous exudative detachment of the retina, usually confined to the macula, and it exhibits a variety of visual manifestations [1]. If CSC lasts more than 3-6 months, it is considered chronic. CSC can also be classified into the classic type and diffused retinal epitheliopathy (DRPE), based on the status of the retinal pigment epithelium (RPE) [2]. The classical type is accompanied with a largely favourable natural history, characterised by a relatively well-preserved retina [2, 3]. DRPE involves extensive RPE disruptions and diffused leakage, leading to a poor prognosis due to anatomical damage. As there are eyes with CSC lasting for more than 6 months but manifesting with recurrent classic characteristics, chronic CSC is not necessarily the same as DRPE. For this reason, we applied this classification to chronic CSC in this study and evaluated only patients presenting with the manifestations of DRPE. DRPE 
a

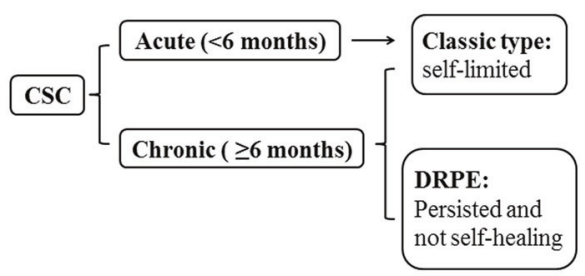

b
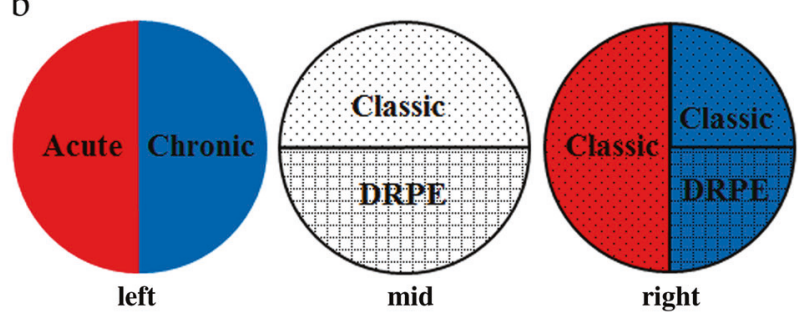

Fig. 1 CSC can be classified to acute or chronic type, based on symptom duration, and can also be subgrouped into classic and DRPE, based on the situation of RPE. In fact, acute type was not always corresponded to classic, as well as the chronic to DPRE. Just as the tree diagram showed, there exists some cases presented with selflimited tendency but recurred lasting for more than 6 months. The widespread RPE decompensation was not witnessed in such recurrent type. The more than 6 months duration was only a necessary but not sufficient condition, while the diffused RPE abnormality was the gold standard to confirm the diagnosis of DRPE. The most common classification of CSC is acute (left, red zone) or chronic type (left, blue zone), 6 months as a dividing point. The situations of RPE can also subgroup CSC into classic (mid, dot zone) and DRPE (mid, grid zone). All acute cases (right, red dot zone) can be categorised to classic type. Recurred cases (right, blue dot zone) with classic character last for more than 6 months, supposed to be classified to chronic CSC. DRPE type (right, blue grid zone) lasts for more than 6 months and presents with widespread RPE decompensation. The pie has no meaning in proportion, but a schematic diagram

represented the specific subtype of chronic CSC in this study, where it largely differed from the classic type due to severe widespread RPE decompensation and a poor prognosis (Fig. 1)

Years ago, photodynamic therapy (PDT) with verteporfin was proposed for treating CSC [4]. In terms of DRPE treatment, a variety of medical treatment modalities have been proposed, including drugs, thermal laser and PDT. However, only PDT has been able to solve the underlying choroidal hyperpermeability problem [5]. The mechanism of action involves halting choriocapillary hyperperfusion in the short term and remodelling the choroidal vascularisation in the long term [6]. However, standard PDT may have irreversible complications when used for treating CSC and DRPE [7, 8]. Modified PDT regimens were found to be associated with improved treatment outcomes and a lower incidence of adverse effects [9-14]. In comparing different regimes, PDT with half-dose verteporfin appeared to be the optimal choice.
Since DRPE presents with long-standing, severe, widespread RPE decompensation, its treatment remains challenging, and the outcomes should be studied [15]. In this research, we aim to evaluate the 24-month efficacy and safety of half-dose PDT for DRPE, guided by simultaneous optical coherence tomography (OCT), fluorescein angiography (FA) and indocyanine green angiography (ICGA).

\section{Methods}

This retrospective study was performed at the Department of Ophthalmology, Eye \& ENT Hospital of Fudan University, Shanghai, China. Patients presenting with chronic DRPE from April 2011 to December 2015 were recruited and followed up for at least 24 months after half-dose PDT administration. All treatments were performed by a retinal specialist (LL).

The patient inclusion criteria were as follows:

1. Visual impairment, central scotoma, metamorphopsia, micropsia, dyschromatopsia or hypermetropisation for $\geq 6$ months;

2. Subretinal fluid (SRF) involving the fovea, as confirmed by OCT, with multiple, widespread or subtle leaks detected by FA, and abnormal choroidal vascular dilation or hyperpermeability detected by ICGA.

The exclusion criteria were as follows:

1. Choroidal neovascularisation (CNV), polyploidal choroidal vasculopathy or other maculopathy;

2. Prior use of corticosteroids in any form;

3. Thermal laser photocoagulation in the last 6 months or history of intravitreal injection of anti-vascular endothelial growth factor agents;

4. Any contraindications for angiographic or verteporfin dyes.

Pre-PDT ocular examinations included measurement of the best-corrected visual acuity (BCVA), using Early Treatment Diabetic Retinopathy Study charts; the outcomes were presented in logarithm of the minimum angle of resolution $(\log$ MAR) scale. Dilated fundus examinations included fundoscopy and slit-lamp biomicroscopy with a 120-D superfield lens. Simultaneous FA, ICGA and OCT were performed using the Spectralis ${ }^{\circ}$ HRA + OCT system (Heidelberg Engineering, Heidelberg, Germany).

To obtain the OCT images, horizontal and vertical linear and volume scans of the fovea and abnormal areas showed on angiography were performed. The external limiting 
membrane (ELM), ellipsoid zone (EZ) and interdigitation zone (IZ) were morphologically classified as absent, fragmented or continuous using the grading scheme proposed by Fujita et al. [16]. The central neural retinal thickness (CNT) was assessed by manually measuring the distance between the internal limiting membrane and photoreceptor outer segments in the central fovea [17, 18].

On FA, diffuse leakage was defined as more than three foci or a widespread active leak, and subtle leakage as nondemonstrable active foci. ICGA was used to assess the two following parameters: choroidal vascular hyperpermeability pre- and post PDT [19] and choriocapillary perfusion post PDT [20]. Each classification was evaluated by YL and LL, who were masked to the patients' characteristics and treatment.

After obtaining written informed consent, $3 \mathrm{mg} / \mathrm{m}^{2}$ of verteporfin was infused over $8 \mathrm{~min}$, followed by laser delivery at $10 \mathrm{~min}$ after the starting infusion. The 689-nm laser (Opal Photoactivator; Lumenis, Beijing, China) was applied for $83 \mathrm{~s}$ at an intensity of $50 \mathrm{~J} / \mathrm{cm}^{2}$, as previously described by Chan et al. [8]. The laser-targeted leakages on FA and choroidal hyperpermeability on ICGA, as well as sites with fluid identified by the Spectralis HRA + OCT system, even without positive manifestations on the angiographs. The spots were intended to cover the greatest linear dimension of the abnormal area while avoiding any overlap on the fovea. An ocular fundus laser lens (Ocular Inc., Bellevue, WA, USA) was used to achieve spot diameters of $1100-5400 \mu \mathrm{m}$. The patients were instructed to avoid light exposure for 5 days post PDT.

All the patients were instructed to attend for follow-up at 0, 3, 6, 12 and 24 months post PDT. BCVA and OCT were analysed at all visits. FA and ICGA were examined at 3 months post PDT. Additional FA and ICGA were carried out if active lesions were persistent or recurrent during the follow-up period.

SPSS software version 20.0 (IBM Inc., Armonk, IL, USA) and GraphPad Prism 6.0 (GraphPad Software Inc., La Jolla, CA, USA) were used for all data analyses and picture constructions. The paired $t$-test, analysis of variance and non-parametric Wilcoxon signed-rank test were used to compare differences. Linear regression analyses were performed to assess the associations between the logMAR BCVA and EZ and IZ. A $p$-value $<0.05$ was considered to indicate statistical significance.

\section{Results}

\section{Patient demographics}

Forty-eight eyes in 48 Chinese patients, 40 males $(83.3 \%)$ and 8 females $(16.6 \%)$, were included in this study. The mean \pm standard deviation age of the patients was $47.8 \pm 7.1$ years (range: 38-66 years). The median duration of symptoms was 21 months (range: 6-120 months). Fourteen eyes (29.2\%) had experienced more than one episode of CSC prior to PDT. The duration of follow-up was 24 months after the initial PDT.

\section{PDT parameters and number of treatment sessions}

The average PDT spot diameter was $3220 \mu \mathrm{m}$ (range: 1100 $5400 \mu \mathrm{m})$. The median number of spots per eye was 2 (range: 1-4). The numbers of spots were 1 in 19 eyes (39.6\%), 2 in 18 eyes (37.5\%) and $\geq 3$ in 11 eyes $(22.9 \%)$. The median number of PDT sessions was one (range: 1-2); 44 eyes $(91.7 \%)$ achieved complete resolution of fluid in one treatment session. Four eyes $(8.3 \%)$ underwent two treatment sessions, among which, 3 eyes (6.3\%) exhibited recurring fluid. All 14 eyes (29.2\%) with a history of symptom recurrence prior to PDT achieved complete resolution of fluids after a single treatment session, and this lasted for the entire follow-up period.

\section{Angiographic findings}

The baseline FA revealed diffuse fluorescein leakage in 36 eyes $(75.0 \%)$ and subtle or oozing leakage in 12 eyes (25.0\%). Middle-phase ICGA revealed intermediate hyperfluorescence in 9 eyes $(18.7 \%)$ and intense hyperfluorescence in 39 eyes $(81.3 \%)$. At 3 months post PDT, there were no signs of leakage in 42 eyes $(87.5 \%)$, subtle leakage in 5 eyes (10.4\%; no serous fluid on OCT) and persistent pigmental epithelial detachment (PED) pooling was observed in 1 eye $(2.1 \%)$. The middle-phase ICGA revealed no hyperfluorescence in 35 eyes $(72.9 \%)$, intermediate hyperfluorescence in 6 eyes $(12.5 \%)$ and intense hyperfluorescence in 7 eyes $(14.6 \%)$.

\section{OCT findings}

At baseline, all eyes (100\%) had SRF involving the central fovea, 3 eyes $(6.25 \%)$ also had intra-retinal fluid (IRF) and 1 eye $(2.1 \%)$ also had extensive PED (affecting an area larger than one entire diameter of the papillary disc). Overall, complete fluid absorption was achieved in 46 eyes $(95.8 \%)$ at 3 months, 45 eyes $(93.8 \%)$ at 6 months, 46 eyes $(95.8 \%)$ at 12 months and 48 eyes $(100.0 \%)$ at 24 months (Fig. 2). Two eyes with isolated SRF were retreated at 6 and 12 months, respectively, due to recurrence of SRF; in both cases, the fluid absorbed at 1 month post PDT. One eye with recurrent SRF and IRF was retreated at 6 months, and the fluid resolved 3 months later. The eye that presented with SRF and PED at baseline had complete resolution of the SRF after the initial treatment. 
Fig. 2 The changes in subretinal fluid and best-corrected visual acuity (BCVA) during whole follow-up $(\mathbf{a}, \mathbf{b})$ resolution of fluid and average BCVA throughout. Bars are the standard error of the mean. The BCVA is significantly better at 6, 12 and 24 months than at baseline. c, d Numbers of integrity of ellipsoid zones (EZ) and interdigitation zones (IZ) on optical coherence tomographic at 3, 6, 12 and 24 months. The numbers of eyes with fragmented and continuous EZ and IZ gradually increased after treatment. In EZ, eyes in group 1 had longer duration than the rest two groups. The rest has no difference between each other. And no statistical difference was found in different groups of IZ (e, f). *Compared with baseline values $p<0.05$
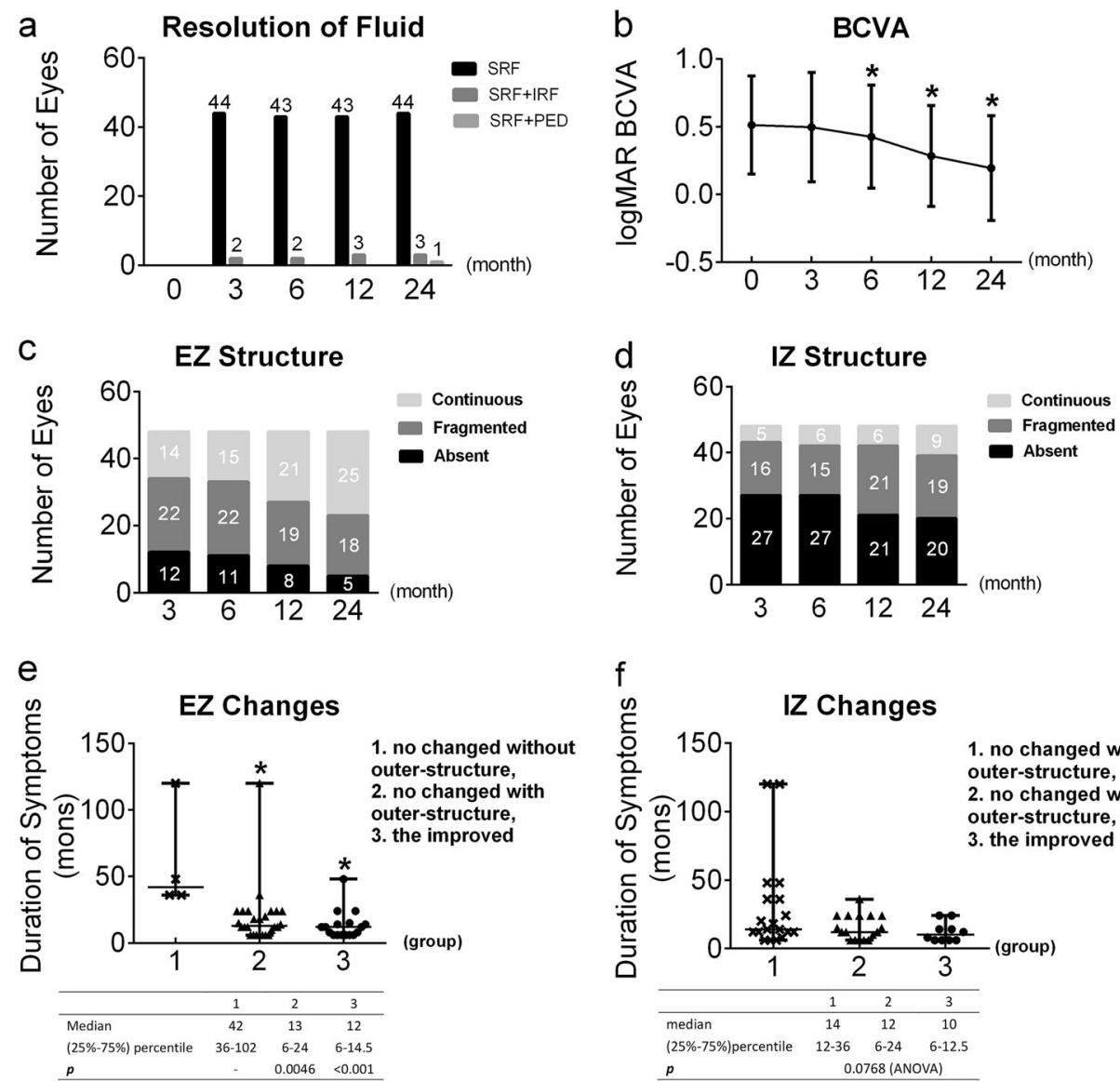

Forty-three eyes $(89.6 \%)$ presented with continuous ELM throughout. Five eyes (10.4\%) presented with fragmented ELM at baseline. Four eyes' ELMs remained fragmented after treatment until 24 months, while the other presented with no changes at 3 months, but it was continuous from 12 months until the final visit. The manifestations of EZ and IZ from 3 to 24 months are shown in Fig. 2. The numbers of eyes in which the EZ or IZ was fragmented or continuous increased gradually during the follow-up period; compared with the 3 months' manifestation, the EZ changed slightly at 6 months $(p=0.414)$; significant structural improvements started at 12 months ( $p$ $=0.008)$ and proceeded to 24 months $(p<0.001)$. The IZ changed slightly at 6 and 12 months $(p=0.705$ and 0.052$)$, and clear recovery emerged up to 24 months $(p=0.012)$. The relation of outer retinal structure changes and durations of symptom were assessed, and found eyes with shorter duration of DRPE reserved the reversibility of EZ (Fig. 2). The CNT remained stable throughout the whole follow-up; it was $116.4 \pm 33.0 \mu \mathrm{m}$ at baseline, 112.6 $\pm 32.7 \mu \mathrm{m}$ at 3 months, $114.0 \pm 31.9 \mu \mathrm{m}$ at 6 months, 115.3 $\pm 32.1 \mu \mathrm{m}$ at 12 months and $118.9 \pm 32.9 \mu \mathrm{m}$ at 24 months $(p>0.05)$.

\section{Changes in visual function}

The baseline BCVA, in logMAR, was $0.51 \pm 0.36$ (range: 0.00 to 1.40 ). The BCVA was unchanged at 3 months, with a mean $\log$ MAR of $0.50 \pm 0.40$ (range: 0.00 to $1.40 ; p=$ 0.336 ). It started to improve to $0.43 \pm 0.38$ (range: -0.18 to $1.40 ; p<0.001)$ at 6 months. The boost continued to $0.29 \pm$ 0.37 (range: -0.2 to $1.10 ; p<0.001$ ) at 12 months and 0.19 \pm 0.39 (range: -0.3 to $1.00 ; p<0.001$ ) at 24 months (Fig. 2). The average improvement in BCVA at the final visit was 3.2 lines. The visual acuity improved by $\geq 2$ lines in 41 eyes (85.4\%) 24 months after treatment, $\geq 4$ lines in 17 eyes $(35.4 \%)$ and no more than 1 line in 7 eyes (14.6\%). None of the eyes experienced a worsening in visual acuity.

During the follow-up, 43 eyes (89.6\%) with continuous ELM had a mean BCVA enhancement for 3.3 lines, while 4 eyes $(8.3 \%)$ with fragmented ELM had an enhancement for 1 line. One eye with fragmented ELM recovered to become continuous, along with obvious improvement for 4 lines (Fig. 3). Similarly, the eyes with a more intact integrity of EZ and IZ possessed better BCVA (Fig. 4). The BCVA in $\log$ MAR was inversely correlated with the EZ and IZ at the $3-, 6-, 12-$ and 24-month visits $(p<0.01)$. 

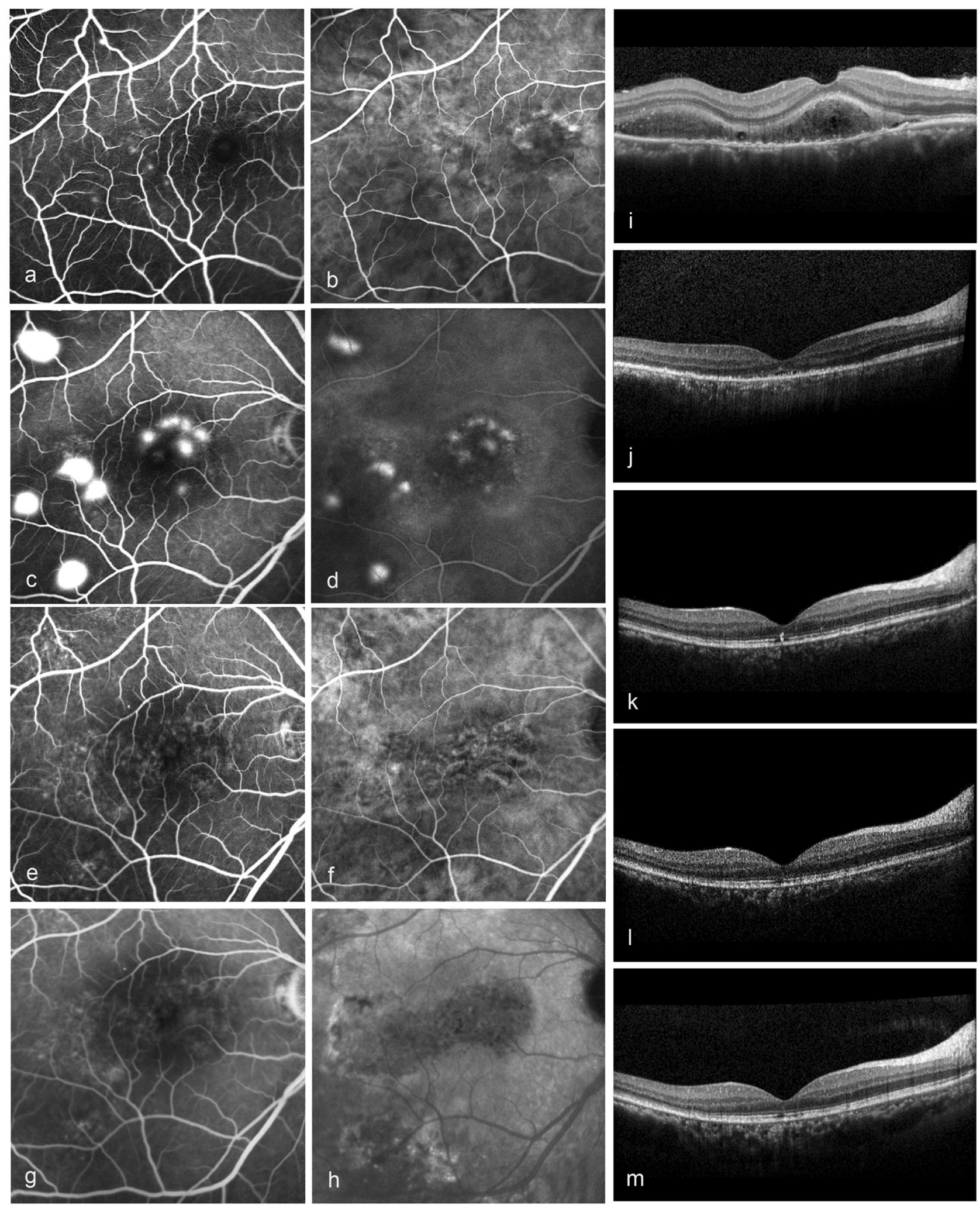

\section{Safety}

None of the patients developed any systemic adverse events due to the verteporfin infusion. At 3 months post PDT,
ICGA revealed mild nonperfusion (grade 1) in 9 (18.8\%) eyes and normal choriocapillary perfusion (grade 0 ) in 39 (81.2\%) eyes. The CNT remained stable during the followup period. There were no cases of further neurosensory 
Fig. 3 A 38-year-old male with diffused retinal pigment epitheliopathy for 14 months and deteriorated over recent 1 month. a, b Early-phase angiography showed diffused hyperfluorescence across the posterior region in fluoroscein angiography (FA) and choroidal vascular dilation in indocyanine green angiography (ICGA). c, d Mid-phase images showed at least 11 active fluorescein leaks and intense choroidal hyperpermeability staining. $\mathbf{e}-\mathbf{h}$ At 3 months, the FA and ICGA images showed no active fluorescein leaks despite the persistent choroidal vascular dilation. Optical coherence tomograpgy images: $\mathbf{i}$ at baseline, external limiting membrane (ELM): fragmented; $\mathbf{j}$ at 3 months, ELM: fragmented, ellipsoid zone (EZ): absent, interdigitation zone (IZ): absent; $\mathbf{k}$ at 6 months, ELM: fragmented, EZ: fragmented, IZ: absent; $\mathbf{l}$ at 12 months, ELM: continuous, EZ: fragmented, IZ: absent; $\mathbf{m}$ at 24 months, ELM: continuous, EZ: fragmented, IZ: fragmented. The subretinal fluid absorbed at 3 months without recurrence, and bestcorrected visual acuity (logarithms of the minimum angle of resolution) was $0.15,0.40,0.22,-0.08$ and -0.20 at each visit

retinal atrophy or secondary $\mathrm{CNV}$ during the follow-up period.

\section{Discussion}

Based on the RPE status, CSC was classified as classic type and DRPE. Classic CSC may occur only once or more than once, but it regresses spontaneously [2,3]. When recurrent symptoms last for more than 6 months, the classic type is regarded as chronic CSC. DRPE, as a more severe type of this disorder, has no inclination for self-healing and always lasts for more than 6 months. Consequently, DRPE belongs to the category of chronic CSC, but chronic CSC is not always identical to DRPE. To specifically exclude the classic type ( $\geq 6$ months) of chronic CSC, we recruited the severest type of chronic CSC.

Since 2003, many studies have evaluated the long-term efficacy of PDT for chronic CSC [5, 17, 21-25]; however, only a few studies have discussed the efficacy and safety of PDT in treating DRPE. We previously reported the favourable outcomes of half-dose PDT for chronic CSC, including the classic type and DRPE [26]. In this study, patients with widespread RPE decompensations were recruited and only 48 eyes met the criterion.

Given the complexity of DRPE, PDT radiation was expected to cover all the active spots to guarantee the efficacy of the treatment. In chronic CSC, active lesions are illustrated from leakage on FA, choroidal hyperfluorescence on ICGA. When it comes to DRPE, except for the classic former two, it should include the sites with fluid demonstrated by the OCT, though uncertainty leaking shown in correspondence areas with angiography [27]. For the fluid comes from active lesion, the PDT laser spot should cover the area. This kind of non-classic active lesions is easy to be missed by a therapist during treatment. Fortunately, the simultaneous FA, ICGA and OCT system allows the interesting stratus on OCT and leaking sites on angiographs,
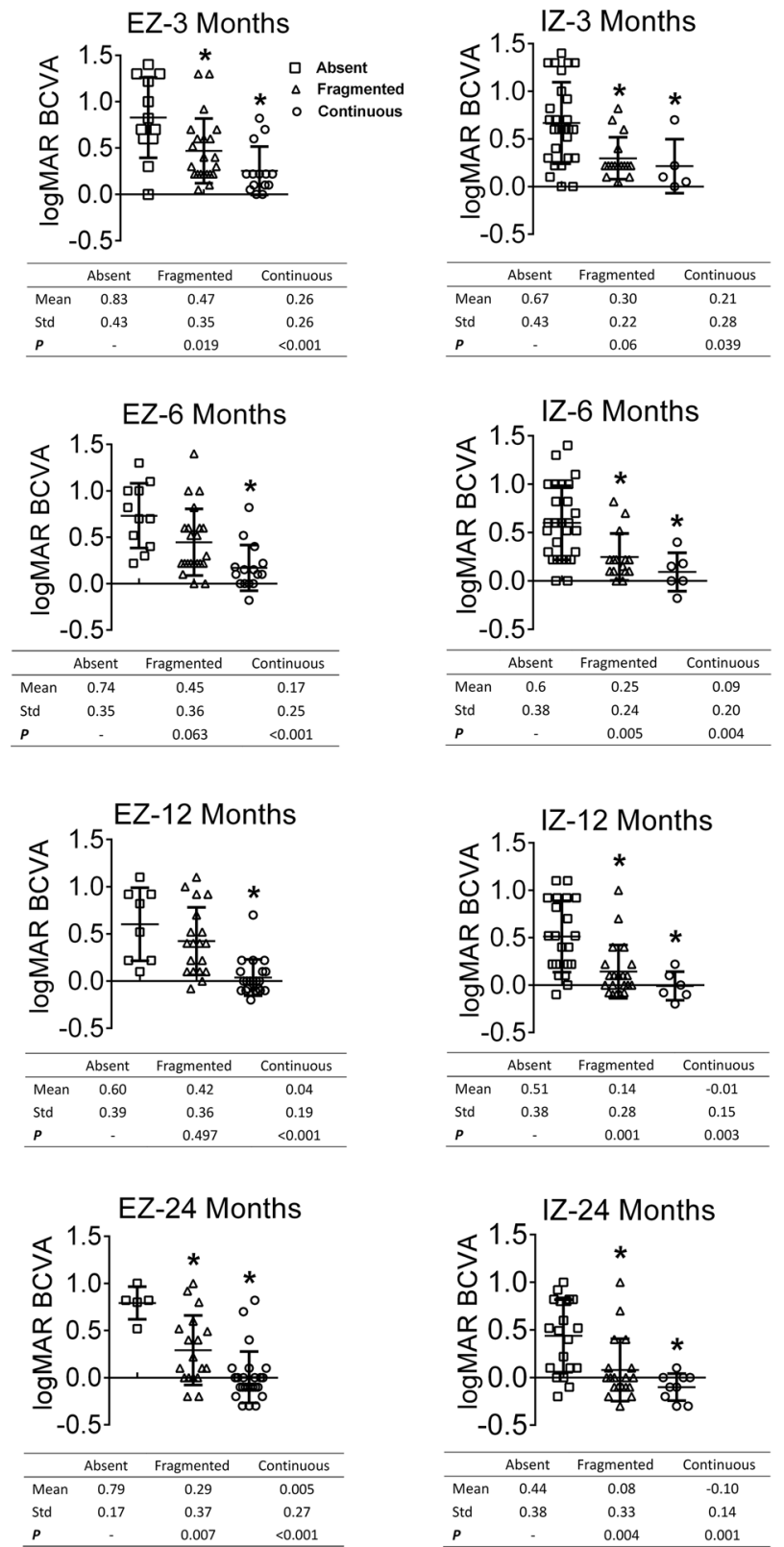

Fig. 4 Scattergram showing the best-corrected visual acuity (BCVA) in logMAR as a function of photoreceptor manifestation on optical coherence tomography after half-dose photodynamic therapy. Note that error bar means variations of BCVA. Different symbols (squares, triangles and circles) represent different status of ellipsoid zones (EZ) and interdigitation zones (IZ). Eyes with more intact integrity of EZ and IZ possessed with better BCVA. *Compared with absent EZ or IZ, $p<0.05$

corresponding to each other, to be visualised [28]. In other words, when the active angiographic manifestation is not clear, a therapist can scan the simultaneous OCT through suspicious leaking sites to determine whether there is fluid. This helps to unveil all active lesions, to prevent them from being missed by a therapist during treatment; as a result, it promotes the resolution of fluid post PDT. In our study, complete fluid absorption was achieved in $95.8 \%$ of eyes at 

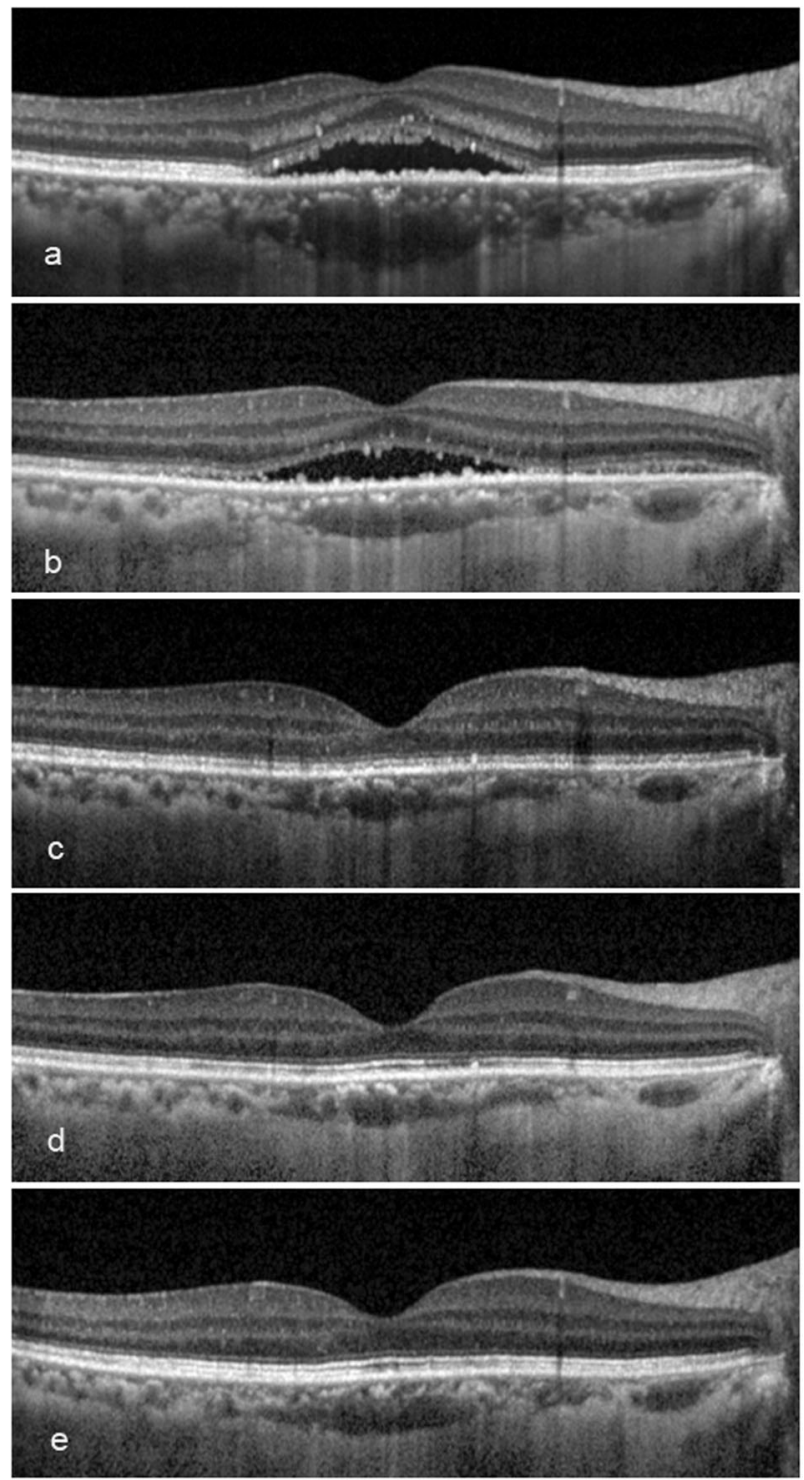

Fig. 5 The outer retinal microstructure recovery post treatment of a 43year-old female with symptom duration for 6 months. a At baseline, ellipsoid zone (EZ): absent, interdigitation zone (IZ): absent; $\mathbf{b}, \mathbf{c}$ at 3 and 6 months, EZ: fragmented, IZ: absent; d at 12 months, EZ: continuous, IZ: absent; e at 24 months, EZ: continuous, IZ: fragmented. The subretinal fluid at baseline had been partly absorbed at 3 months, and completely absorbed at 6 months. The external limiting membrane presented continuous along the whole follow-up. The best-corrected visual acuity (logarithms of the minimum angle of resolution) was $0.22,0.10,0.00,-0.10$ and -0.30 at each visit

3 months and $100.0 \%$ of eyes at 24 months. The recurrence rate was $6.3 \%$.

After the resolution of fluid, the recovery of the microstructure on OCT and BCVA represented major indicators reflecting the efficacy of half-dose PDT. The ELM, EZ and $\mathrm{IZ}$, the common indicators of the microstructure $[15,16$, 29], were also used to assess the microstructural recovery (Fig. 2). As the changes in the ELM were slight throughout the follow-up, only the improvement of BCVA on different
ELM statuses was compared. Gutiérrez-Hernández et al. [29] previously suggested that an intact ELM could forecast the possibility of visual recovery. We also found that eyes with continuous ELM gained greater improvements. One eye with a fragmented ELM at baseline recovered with an apparent BCVA benefit (Fig. 3). Although we did not find that every injured ELM could still regenerate post half-dose PDT, this specific reappearance of the ELM may predict the likelihood of BCVA recovery.

The baseline morphologies of EZ and IZ were not evaluated because of the presence of fluid. By comparing the morphologies at each visit, it was evident that the integrities of the EZ and IZ improved. Only in EZ, shorter duration appeared to reserve the ability to reversibility (Fig. 2). A longer follow-up and larger sample size study may be needed to confirm the non-significance in IZ changes. The BCVA in logMAR was better in eyes with more intact EZ and IZ structures (Fig. 4). Based on the regression analyses, the improvements in function seem to have been due to the renewed structural integrities (Fig. 5).

The average BCVA remained unchanged from baseline to 3 months, but it started to improve significantly at 6 months. The occurrence of BCVA improving appeared later than in other studies [16, 21-25], which implied that the recruited patients manifested more severe baseline conditions than are apparent in the general chronic CSC, and thus, they required a longer period to recover. The improvement continued until 24 months, confirming the efficacy of simultaneous, ICGA and OCT-guided PDT.

Secondary CNV and neural retinal atrophy are the severest side effects of full-dose PDT. Previous research has established that choroidal hypoperfusion increases the risk of developing CNV [5, 6, 8, 30, 31]. Our results demonstrated that, at 3 months, only a mild nonperfusion effect (grade I) was observed in $16.7 \%$ of eyes, and none of the patients had developed CNV by the 24-month follow-up visit. The CNT was evaluated to reflect the condition of the neural retina. We applied Spectralis ${ }^{\circ}$ HRA + OCT, which can automatically scan the same retinal stratus at different visits, and found that the CNT remained stable until 24 months.

The limitations of this study included the small sample size, retrospective design and lack of control group. In future investigation, other examinations, including OCT angiography, multifocal electroretinography and microperimetry could provide further valuable information on the efficacy of the treatment. Researchers could also consider assessing the morphological and functional states of the choroid.

\section{Conclusion}

Simultaneous FA, ICGA and OCT facilitated more comprehensive guidance for half-dose PDT treatment in all 48 
eyes with DRPE, ensuring its efficacy and safety meantime. The BCVA improvement, which emerged at 6 months, later than general chronic CSC, appeared to be attributable to the restoration of the outer retinal structure.

\section{Summary}

\section{What was known before}

- Since 2003, many studies have evaluated the long-term favourable efficacy of PDT for chronic CSC.

\section{What this study adds}

- However, only a few studies have discussed the efficacy and safety of PDT in treating DRPE. In this study, only patients with widespread RPE decompensation were recruited. In eyes with DRPE, simultaneous FA, ICGA and OCT facilitated more comprehensive guidance for half-dose PDT treatment, ensuring its efficacy and safety. Although the visual acuity improved at 6 months, latter than other studies, it did happen, along with the recovery of retinal structure.

\section{Compliance with ethical standards}

Conflict of interest The authors declare that they have no conflict of interest.

Open Access This article is licensed under a Creative Commons Attribution 4.0 International License, which permits use, sharing, adaptation, distribution and reproduction in any medium or format, as long as you give appropriate credit to the original author(s) and the source, provide a link to the Creative Commons license, and indicate if changes were made. The images or other third party material in this article are included in the article's Creative Commons license, unless indicated otherwise in a credit line to the material. If material is not included in the article's Creative Commons license and your intended use is not permitted by statutory regulation or exceeds the permitted use, you will need to obtain permission directly from the copyright holder. To view a copy of this license, visit http://creativecommons. org/licenses/by/4.0/.

\section{References}

1. Gass JD. Pathogenesis of disciform detachment of the neuroepithelium. Am J Ophthalmol. 1967;63:S1-139.

2. Nicholson B, Noble J, Forooghian F, Meyerle C. Central serous chorioretinopathy: update on pathophysiology and treatment. Surv Ophthalmol. 2013;58:103-26.

3. Pang CE, Shah VP, Sarraf D, Freund KB. Ultra-Widefield imaging with autofluorescence and indocyanine green angiography in central serous chorioretinopathy. Am J Ophthalmol. 2014;158:362-71.e2.

4. Treatment of age-related macular degeneration with photodynamic therapy (TAP) Study Group. Photodynamic therapy of subfoveal choroidal neovascularization in age-related macular degeneration with verteporfin: one-year results of 2 randomized clinical trials--TAP report. Arch Ophthalmol. 1999;117:1329-45.

5. Rouvas A, Stavrakas P, Theodossiadis PG, Stamatiou P, Milia M, Giannakaki E, et al. Long-term results of half-fluence photodynamic therapy for chronic central serous chorioretinopathy. Eur J Ophthalmol. 2012;22:417-22.

6. Chan WM, Lam DS, Lai TY, Tam BS, Liu DT, Chan CK. Choroidal vascular remodelling in central serous chorioretinopathy after indocyanine green guided photodynamic therapy with verteporfin: a novel treatment at the primary disease level. $\mathrm{Br} \mathbf{J}$ Ophthalmol. 2003;87:1453-8.

7. Cardillo Piccolino F1, Eandi CM, Ventre L, Rigault de la Longrais RC, Grignolo FM. Photodynamic therapy for chronic central serous chorioretinopathy. Retina. 2003;23:752-63.

8. Colucciello M. Choroidal neovascularization complication photodynamic therapy for central serous retinopathy. Retina. 2006;26:239-42.

9. Chan WM, Lai TY, Lai RY, Tang EW, Liu DT, Lam DS. Safety enhanced photodynamic therapy for chronic central serous chorioretinopathy: one-year results of a prospective study. Retina. 2008;28:85-93.

10. Zhao M, Zhang F, Chen Y, Dai H, Qu J, Dong C, et al. A 50\% vs $30 \%$ dose of verteporfin (photodynamic therapy) for acute central serous chorioretinopathy: one-year results of a randomized clinical trial. JAMA Ophthalmol. 2015;13:333-40.

11. Reibaldi M, Cardascia N, Longo A, Furino C, Avitabile T, Faro S, et al. Standard-fluence versus low-fluence photodynamic therapy in chronic central serous chorioretinopathy: a nonrandomized clinical trial. Am J Ophthalmol. 2010;149:307-15.

12. Butler AL, Fung AT, Merkur AB, Albiani DA, Forooghian F. Very minimal fluence photodynamic therapy for chronic central serous chorioretinopathy. Can J Ophthalmol. 2012;47:42-4.

13. Liu CF, Chen LJ, Tsai SH, Lai CC, Chan WC, Wu WC, et al. Half-dose verteporfin combined with half-fluence photodynamic therapy for chronic central serous chorioretinopathy. J Ocul Pharmacol Ther. 2014;30:400-5.

14. Nicoló M, Eandi CM, Alovisi C, Grignolo FM, Traverso CE, Musetti D, et al. Half-fluence versus half-dose photodynamic therapy in chronic central serous chorioretinopathy. Am J Ophthalmol. 2014;157:1033-7.

15. Tsakonas GD, Kotsolis AI, Koutsandrea C, Georgalas I, Papaconstantinou D, Ladas ID. Multiple spots of photodynamic therapy for the treatment of severe chronic central serous chorioretinopathy. Clin Ophthalmol. 2012;6:1639-44.

16. Fujita K, Shinoda K, Imamura Y, Matsumoto CS, Mizutani Y, Mizota A, et al. Correlation of integrity of cone outer segment tips line with retinal sensitivity after half-dose photodynamic therapy for chronic central serous chorioretinopathy. Am J Ophthalmol. 2012;154:579-85.

17. Matsumoto H, Kishi S, Otani T, Sato T. Elongation of photoreceptor outer segment in central serous chorioretinopathy. Am J Ophthalmol. 2008;145:162-8.

18. Silva RM, Ruiz-Moreno JM, Gomez-Ulla F, Montero JA, Gregório T, Cachulo ML, et al. Photodynamic therapy for chronic central serous chorioretinopathy: a 4-year follow-up study. Retina. 2013;33:309-15.

19. Inoue R, Sawa M, Tsujikawa M, Gomi F. Association between the efficacy of photodynamic therapy and indocyanine green angiography findings for central serous chorioretinopathy. Am J Ophthalmol. 2010;149:441-6.

20. Michels S, Hansmann F, Geitzenauer W, Schmidt-Erfurth U. Influence of treatment parameters on selectivity of verteporfin therapy. Invest Ophthalmol Vis Sci. 2006;47:371-6.

21. Vasconcelos H, Marques I, Santos AR, Melo P, Pires I, Figueira J, et al. Long-term chorioretinal changes after photodynamic therapy 
for chronic central serous chorioretinopathy. Graefes Arch Clin Exp Ophthalmol. 2013;251:1697-705.

22. Karakus SH, Basarir B, Pinarci EY, Kirandi EU, Demirok A. Long-term results of half-dose photodynamic therapy for chronic central serous chorioretinopathy with contrast sensitivity changes. Eye. 2013;27:612-20.

23. Lai FH, Ng DS, Bakthavatsalam M, Chan VC, Young AL, Luk FO, et al. A multicenter study on the long-term outcomes of halfdose photodynamic therapy in chronic central serous chorioretinopathy. Am J Ophthalmol. 2016;170:91-9.

24. Cheng C, Chang C, Peng C. Comparison of photodynamic therapy using half-dose of verteporfin or half-fluence of laser light for the treatment of chronic central serous chorioretinopathy. Retina. 2017;37:325-33.

25. Tseng $\mathrm{C}$, Chen S. Long-term efficacy of half-dose photodynamic therapy on chronic central serous chorioretinopathy. Brit J Ophthalmol. 2015;99:1070-7.

26. Liu Y, Li L, Xu G, Wang W. Observation on long-term efficacy of half-dose photodynamic therapy with chronic central serous chorioretinopathy using optical coherence tomography. Zhonghua Yan Ke Za Zhi. 2016;52:328-34.
27. Iida T, Kishi S, Hagimura N, Shimizu K. Persistent and bilateral choroidal vascular abnormalities in central serous chorioretinopathy. Retina. 1999;19:508-12.

28. Mathew R, Richardson M, Sivaprasad S. Predictive value of spectral-domain optical coherence tomography features in assessment of visual prognosis in eyes with neovascular agerelated macular degeneration treated with ranibizumab. Am J Ophthalmol. 2013;155:720-6.e1.

29. Gutiérrez-Hernández JC, Martínez-Camarillo JC, Sadda SR. Long-term follow-up of photoreceptor loss and recovery after half-fluence photodynamic therapy for chronic central serous chorioretinopathy. Retin Cases Brief Rep. 2015;9:109-13.

30. Lee PY, Kim KS, Lee WK. Severe choroidal ischemia following photodynamic therapy for pigment epithelial detachment and chronic central serous chorioretinopathy. Jpn J Ophthalmol. 2009;53:52-6.

31. Copete S, Ruiz-Moreno JM, Cava C, Montero JA. Retinal thickness changes following photodynamic therapy in chronic central serous chorioretinopathy. Graefes Arch Clin Exp Ophthalmol. 2012;250:803-8. 